\title{
EFFETS SPATIO-TEMPORELS DE LA REFLEXION ET DE LA RÉFRACTION PAR UNE LAME À FACES PARALLÈLES IMMERGÉE
}

\author{
P. CHEVÉE et M. DESCHAMPS \\ Laboratoire de Mécanique Physique, Université de Bordeaux I, URA C.N.R.S. $N^{\circ} 867,351$ Cours de \\ la Libération, F-33405 Talence cedex, France
}

\begin{abstract}
In the framework of linear acoustics, the diffraction effects of a field issued from a transducer with narrow aperture through an immersed solid plate, are studied. A numerical calculation using spatial Fourier analysis has been performed to obtain its evolution under harmonic, and pulsed excitation. Both isotropic and anisotropic cases are treated, taking into account the viscoelasticity, or not. Theoretical results obtained for particular materials are compared with the experience.
\end{abstract}

\section{Introduction}

Les effets de diffraction en régime entretenu d'un faisceau acoustique borné au passage d'un ou de plusieurs dioptres sont abondamment décris dans la littérature. Ces études font appel à différents outils mathématiques tels que l'analyse de Fourier, la représentation de Green du champ, ou la décomposition en ondes planes hétérogènes. Pour ces trois méthodes, voir par exemple les références[1 à 3] respectivement. L'objet de cet article est de reprendre le cas de la diffraction d'un champ par une lame à faces parallèles en régime impulsionnel, et ce, pour un matériau viscoélastique anisotrope ou non. Signalons qu'une précédente étude en régime entretenu pour un tel type de matériau a récemment été entreprise [4], et que le cas de la transmission en régime impulsionnel à travers une plaque viscoélastique en incidence normale a aussi été traité [5]. Afin de valider les résultats numériques obtenus, une confrontation avec l'expérience a été effectuée.

\section{1) Formulation du problème}

Considérons une lame anisotrope d'épaisseur finie et d'extension infinie, immergée dans un fluide parfait initialement au repos, et susceptible de pivoter d'un angle azimuthal $\psi$. Un traducteur émet un champ acoustique borné dans le temps et dans l'espace, qui vient insonner la lame sous une incidence $\theta$ et est recueilli par un traducteur récepteur décalé latéralement d'une distance $\mathrm{x}$, et distant de $\mathrm{z}$ par rapport à l'émetteur.

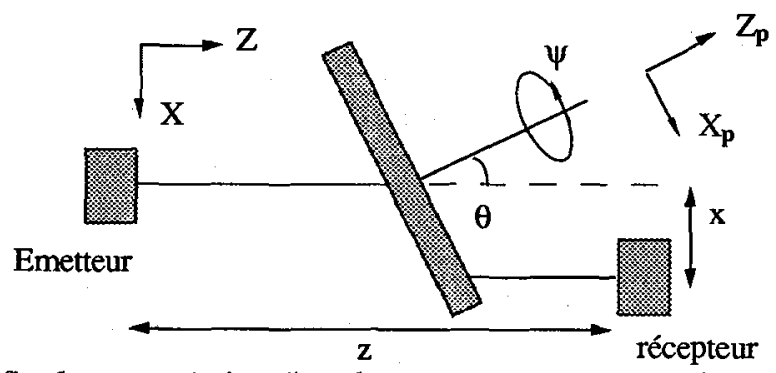

fig. 1: transmission d'un champ acoustique par une lame $a$ faces paralleles immergée. 
Si nous définissons le spectre $\Phi$ du signal d'excitation comme étant la transformée de Fourier directe par rapport au temps, et inverse par rapport à l'espace, le champ potentiel recueilli est donné par l'intégrale:

$$
\varphi(x, z, t)=\iint_{-\infty}^{+\infty} \Phi(u, f, z=0) H(u, f, \psi, z) e^{2 i \pi(f t-u x) d u d f}
$$

où $x$ est la position du récepteur par rapport à l'axe central de l'émetteur, $t$ le temps, $f$ la fréquence temporelle, $u$ la fréquence spatiale suivant $X, \Phi$ le spectre du signal initial, et $H$ la fonction de transfert de l'ensemble (eau, plaque). Le choix de la dernière transformée inverse ne s'impose pas, mais permet l'écriture du champ sous la forme classique d'une somme d'ondes planes homogènes progressives [6]. La fonction de transfert $H$ peut s'écrire comme le produit d'un facteur relatif à la propagation pure dans le fluide de couplage, et d'un facteur relatif à la traversée de l'échantillon:

$$
H(u, f, z)=\exp [-i 2 \pi w z] T\left(C_{i j}, \eta_{i j}, \psi, f, u_{p}\right),
$$

où la fonction $\mathrm{T}$ est le coefficient de transmission de la lame, qui rend compte de l'excitation des différents modes dans le solide, de leurs multiple conversions aux interfaces et de leurs interférences. En toute généralité, $\mathrm{T}$ est fonction des caractéristiques du matériau, c'est à dire des constantes d'élasticité $\mathrm{C}_{\mathrm{ij}}$ et de viscoélasticité $\eta_{\mathrm{ij}}$, de la fréquence $\mathrm{f}$, de l'angle azimuthal si le matériau est anisotrope, et de la fréquence spatiale $u_{\mathrm{p}}$ dans la direction $X_{\mathrm{p}}$, laquelle correspond à l'angle d'incidence de chaque mode plan:

$$
u_{p}=u \cos \theta-w \sin \theta \text {. }
$$

La fréquence spatiale w suivant la direction $\mathrm{Z}$ est couplée à u et à f par l'équation de dispersion:

$$
u^{2}+w^{2}-f^{2} / c^{2}=0,
$$

où $\mathrm{c}$ est la célérité dans le fluide. Signalons que, dans le but d'éviter les phénomènes de repliement de spectre et de signal inhérents à l'emploi d'algorithmes de calcul discret, l'évaluation de T est effectuée par la méthode des séries de Debye, dont on trouvera une description détaillée en [7] pour les matériaux isotropes, et en [8] pour les matériaux anisotropes. L'intérêt d'une telle méthode, valable surtout en régime impulsionnel, est de pouvoir choisir explicitement le nombre de paires ou de triplets d'échos quasi longitudinal-quasi transversaux transitant dans le matériau.

\section{2) Régime entretenu}

Le champ initial d'excitation à une fréquence $\mathrm{f}=$ fo est modélisé par une gaussienne centrée sur l'axe de l'émetteur, dont l'évaluation de la largeur à mi-hauteur b est effectuée empiriquement par comparaison avec l'allure du signal propagé sans échantillon.

$$
\Phi(u, f, z=0)=b \exp \left[-\pi b^{2} u^{2}\right] \delta\left(f-f_{o}\right)
$$

Dans ces conditions, le champ $\varphi$ est donné par une intégrale simple par rapport à u. La figure $2 \mathrm{a}$ illustre l'évolution de $\varphi(x, z, t)$ en fonction de la position $x$ du récepteur pour une lame d'aluminium d'épaisseur $9,83 \mathrm{~mm}$, à une incidence $\theta=25^{\circ}$ et à la fréquence $\mathrm{f}_{\mathrm{O}}=1,003 \mathrm{MHz}$ en régime entretenu. La masse volumique vaut $2,78 \mathrm{mg} / \mathrm{mm}^{3}$, la célérité longitudinale vaut $\mathrm{Cl}=6400 \mathrm{~m} / \mathrm{s}$ et la célérité transversale $\mathrm{Ct}=3120 \mathrm{~m} / \mathrm{s}$. La distance $\mathrm{z}$ est de $320 \mathrm{~mm}$, et la largeur à mi-hauteur b est ajustée à $20 \mathrm{~mm}$. Il est à remarquer le bon accord entre la théorie et l'expérience, qui valide ainsi le choix de la fonction d'ouverture. La présence de plusieurs lobes caractérise, pour cet angle de $25^{\circ}$, $1^{\prime}$ existence d'interférences tour à tour constructives et destructives au sein de la lame. La figure $2 \mathrm{~b}$ illustre le cas l'une lame de Carbone-Epoxy orthotrope et viscoélastique, d'épaisseur $4,434 \mathrm{~mm}$ et de masse volumique $1,5 \mathrm{mg} / \mathrm{mm}^{3}$, pour une 
fréquence $\mathrm{f}_{\mathrm{O}}=2,242 \mathrm{MHz}$. Dans le but d'évaluer les effets dûs à l'atténuation, l'évolution du champ en fonction de $\mathrm{x}$ a aussi été calculée sans prendre en compte la viscoélasiticité du matériau, et est présentée par une courbe en pointillés. Les constantes d'élasticité $C_{\mathrm{ij}}$ et de viscoélasticité $\eta_{\mathrm{ij}}$ de ce matériau sont présentées dans le tableau 1. Il faut noter que ces derniers coefficients dépendent étroitement de la fréquence; ici ils ont été mesurés à la fréquence $\mathrm{f}_{\mathrm{m}}=\mathrm{f}_{\mathrm{o}}$.

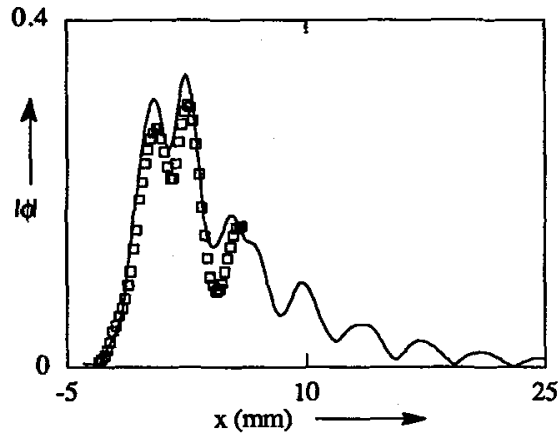

Fig. 2a: profil du faisceau transmis a $\theta=25^{\circ}$. - Théorie uExpérience.

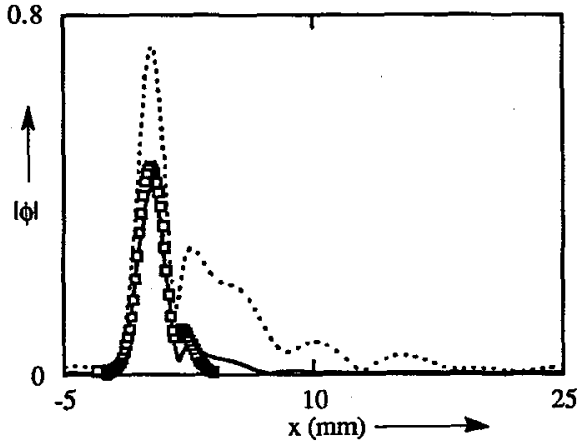

Fig. 2b: profil du faisceau transmis à $\theta=8^{\circ}, \psi=90^{\circ}$. - Théorie (viscoélastique)

.Théorie (non viscoélastique) ם Expérience

Tableau 1. Constantes d'élasticité et de viscoélasticité du Carbone-Epoxy

constantes d'élasticité en GPa.

\begin{tabular}{|c|c|c|c|c|c|c|c|c|}
\hline $\mathrm{C}_{11}^{0}$ & $\mathrm{C}_{22}^{0}$ & $\mathrm{C}_{33}^{0}$ & $\mathrm{C}_{12}^{0}$ & $\mathrm{C}_{13}^{0}$ & $\mathrm{C}_{23}^{0}$ & $\mathrm{C}_{44}^{0}$ & $\mathrm{C}_{55}^{0}$ & $\mathrm{C}_{66}^{0}$ \\
\hline 12.1 & 12.3 & 132 & 5.5 & 5.9 & 6.9 & 6.15 & 6.21 & 3.32 \\
\hline
\end{tabular}
\begin{tabular}{|c|c|c|c|c|c|c|c|c|}
\hline$\eta_{11}$ & $\eta_{22}$ & $\eta_{33}$ & $\eta_{12}$ & $\eta_{13}$ & $\eta_{23}$ & $\eta_{44}$ & $\eta_{55}$ & $\eta_{66}$ \\
\hline 0.043 & 0.037 & 0.4 & 0.021 & 0.016 & 0.001 & 0.02 & 0.015 & 0.009 \\
\hline
\end{tabular}

\section{3) Régime impulsionnel}

Conformément à l'hypothèse classique de découplage du champ initial à la surface de l'émetteur, le signal d'excitation est obtenu en multipliant un signal temporel gaussien, de fréquence centrale $f_{\mathfrak{c}}$ et de largeur à mi-hauteur $\tau$, par la fonction d'ouverture du traducteur décrite au chapitre 2 . Le spectre $\Phi$ peut donc s'écrire:

$$
\Phi(u, f)=A(u) E(f),
$$

où $E(f)$ est le spectre de la partie temporelle pure du champ incident. La formule (2) impliquant aussi la connaissance des viscoélasticités en fonction de la fréquence, l'introduction d'un modèle simple valable dans la bande utilisée s'est avérée probante:

$$
\eta_{\mathrm{ij}}=\eta_{\text {oij }} \mathrm{f}_{\mathrm{m}} / \mathrm{f} \text {, }
$$

où $\eta_{\text {oij }}$ sont les coefficients de viscoélasticité mesurés à la fréquence $f_{m}$. Ce modèle a été vérifié expérimentalement pour le Carbone-Epoxy [8].

Les figures $3 a$ et $3 b$ illustrent, pour les échantillons décrits plus haut, l'allure d'un signal bref transmis. Les paramètres requis pour la simulation sont les mêmes que précédemment, avec, de plus, 
$\mathrm{f}_{\mathrm{c}}=2,148 \mathrm{MHz}$ et $\tau=0,6 \mu \mathrm{s}$; ces derniers dépendent étroitement de la nature des traducteurs. Le nombre d'échos choisi est 5 . On remarque sur la figure 3a (cas de la plaque d'aluminium) que l'écho de plus forte amplitude n'est pas l'écho spéculaire, comme cela aurait été le cas pour $\mathrm{x}=0 \mathrm{~mm}$.
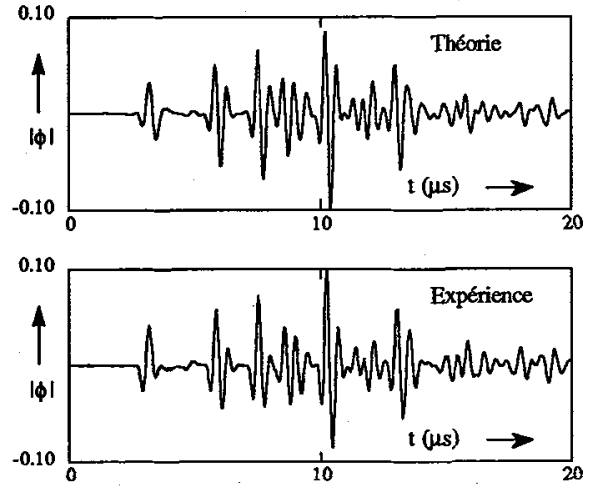

Fig.3a: allure d'un signal transmis a $6^{\circ}$ dans l'aluminium pour $x=-23 \mathrm{~mm}$.

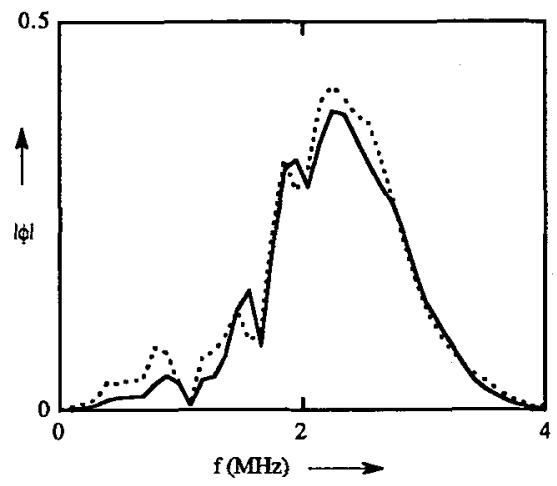

Fig. 3b: spectre du signal transmis a $\theta=6^{\circ}$, $\psi=90^{\circ}$ dans le $C$-Epoxy, pour $x=9,375 \mathrm{~mm}$. -Théorie (viscoélastique) .. Expérience

\section{Conclusion}

Le bon accord entre les résultats théoriques et expérimentaux valide, d'une part, l'emploi de l'analyse de Fourier, et d'autre part, les différents modèles employés pour simuler le champ d'excitation et l'amortissement dans le matériau. Il est cependant possible que ce dernier ait besoin d'être affiné, surtout aux basses fréquences. Une étude précédente [4] a montré en particulier que les effets d'ouverture finie étaient négligeables vis-à-vis de l'atténuation. En revanche, bien que l'emploi des séries de Debye s'est avéré efficace, il n'en demeure pas moins des problèmes de convergence liés aux angles critiques.

\section{Références bibliogranhiques}

[1] Goodman J.W. , "Introduction à l'optique de Fourier et à l'holographie", Masson, Paris, 1972.

[2] Stepanischen P. R. "Transient radiation from pistons in infinite planar baffle", J. Acous. Soc. Am. 70 (1981), 186-204.

[3] Claeys J.M. and Leroy O., "Reflection and transmission of bounded sound beams on half-spaces and through plates", J.Acoust.Soc.Am., 72 (2),(1982), 585-590.

[4] Chevée $P$. and Deschamps $M$. "Non specular effects of reflection and transmission at an immersed viscoelastic - anisotropic layer". Ultrasonics International 91, Le Touquet, France.

[5] Pialucha T., Guyott C.C.H. and Cawley P. "Amplitude spectrum method for measurement of phase velocity". Ultrasonics 27 (1989), 270-279.

[6] Deschamps M. et Roux J. "Effet d'ouverture spatio-temporelle au passage d'un dioptre plan", J.Acoustique 1, (1988), 81-87.

[7] Deschamps M. and Cao C. "Reflection/refraction of a solid layer by Debye's series expansion", Ultrasonics, 29, (1991), 288-293.

[8] Deschamps M. and Hosten B. "The effects of viscoelasticity on the reflection and transmission of ultrasonic waves by an orthotropic plate". Will be published in J.Acoust.Soc.Am. 